\title{
Expectativas académicas y laborales en estudiantes de enfermería
}

\author{
Academic and labor expectations for nursing students \\ Expectativas acadêmicas e de trabalho em estudantes de enfermagem \\ Damariz Escobar* \\ Esmeralda Covarrubias ${ }^{* *}$
}

\section{Resumen}

Objetivo: Conocer las expectativas académicas y laborales de estudiantes que cursan el último semestre de la licenciatura en enfermería, en una universidad pública de la Ciudad de México. Materiales y método: Estudio cuantitativo, descriptivo- exploratorio y transversal. La población de estudio se integró por 492 estudiantes, con una muestra de 105 universitarios, mayores de 18 años, de ambos sexos e inscritos en el último semestre de alguna de las dos carreras que se ofertan en la institución: Licenciatura en Enfermería y Licenciatura en Enfermería y Obstetricia. Para la recolección de datos se utilizó un diseño de muestreo probabilístico aleatorio simple y se elaboró un instrumento autoadministrado de preguntas cerradas, que se sometió a la validación de contenido por parte de dos expertos. Se calcularon frecuencias relativas y absolutas. Resultados: Los estudiantes esperan realizar su servicio social en áreas clínicas, titularse por medio de estudios de posgrado y realizar una especialidad. Sus expectativas profesionales se enfocan en trabajar en el sector público, en hospitales de segundo y tercer nivel de atención; igualmente, quieren ingresar al campo laboral con la categoría de enfermero especialista durante los primeros seis meses después de liberar el servicio social. Conclusiones: Las expectativas académicas y profesionales de los estudiantes se centran en el área asistencial sin considerar el primer nivel de atención, el ejercicio libre de la profesión ni las áreas de docencia, gestión e investigación.

Palabras clave: Estudiantes de enfermería, educación en enfermería, expectativas académicas, expectativas laborales

\section{Abstract}

Objective: To know the academic and labor expectations of students in their last semester of Nursing school in a public university in Mexico City. Materials and method: Quantitative study, descriptive-exploratory and cross-sectional. The study population were 492 students, with a sample of 105 university students, older than 18 , of both sexes and enrolled in their last semester of any of the two degrees offered in the institution: Nursing bachelor's degree and Nursing and Obstetrics bachelor's degree. For the recollection of data, a simple random probability sampling design

\section{Autor de correspondencia}

* $\triangle$ Estudiante de la Maestría en Medicina Social en la Universidad Autónoma Metropolitana- Xochimilco (UAM-X), Licenciada en Enfermería y Obstetricia por la Escuela Nacional de Enfermería y Obstetricia de la Universidad Nacional Autónoma de México (ENEO-UNAM). Correo: vdamariz. edr@hotmail.com. Orcid: https://orcid. org/0000-0002-9003-820X Ciudad de México, México.

** Doctora en Ciencias por el Centro de Investigación y de Estudios Avanzados (CINVESTAV), Maestra en Medicina Social por la UAM-X, Licenciada en Enfermería y Obstetricia por la ENEO- UNAM. Profesora Investigadora del Departamento de Atención a la Salud, UAM-X. Correo: esmeralda-cl@ hotmail.com. Orcid: https://orcid.org/00000001-9161-307X Ciudad de México, México. 
ISSN-PRINT

1794-9831

E-ISSN 2322-7028

Vol. 16 No. 2

May - Ago 2019

Cúcuta, Colombia

was used, and a self-administered instrument of closed questions was used, which was submitted for the validation of its content by two experts. Relative and absolute frequencies were calculated. Results: The students expect to do their social service in clinical areas, earn their degree through postgraduate studies and obtain a specialization. Their professional expectations are focused in working with the public sector in hospitals of second and third levels of care; also, they wish to get into the labor field with the category of nurse practitioner within the first six months after finishing social service. Conclusions: The academic and professional expectations of the students are centered on the care area without considering the primary level of care, the free professional practice, and the areas of teaching, management and research.

Keywords: Nursing students, nursing education, academic expectations, labor expectations.

\section{Resumo}

Objetivo: Conhecer as expectativas acadêmicas e de trabalho de alunos que cursam o último período da licenciatura em enfermagem de uma universidade pública da cidade do México. Materiais e métodos: Estudo quantitativo, descritivo-exploratório e transversal. A população estudada esteve composta por 492 alunos e uma amostra de 105 alunos matriculados no último período da graduação em licenciatura em enfermagem e licenciatura em enfermagem e obstetrícia, com idades maiores de 18 anos e de ambos os sexos. Para a coleta dos dados utilizou-se a técnica de amostragem probabilística aleatória simples e empregou-se um questionário com perguntas fechadas que foi previamente validado por dois expertos. Foram calculadas frequências relativas e absolutas. Resultados: Os estudantes esperam desenvolver seu serviço social na área hospitalar, obter formação de pós-graduação lato sensu e virar especialistas. Suas expectativas de trabalho focam-se no setor público em hospitais das clínicas, além disso, querem ingressar no campo de trabalho como enfermeiros especialistas logo após 6 meses de terem terminado o serviço social. Conclusão: As expectativas acadêmicas e profissionais dos alunos centram-se na área assistencial sem considerar a atenção primária à saúde, docência, administração e pesquisa.

Palavras-chave: Estudantes de enfermagem, educação em enfermagem, expectativas acadêmicas, expectativas de trabalho.

\section{Introducción}

Hasta finales del siglo XX se reconocía a la universidad como la institución que formaba recursos humanos para favorecer el desarrollo industrial y de servicios (1). Actualmente, el fin central del proceso de educación profesional es que, al terminar sus estudios, los universitarios obtengan un trabajo bien remunerado que les permita realizarse personalmente y cubrir sus necesidades esenciales (2). Aquí es donde cobra sentido la relación entre el sistema educativo y el mercado laboral. Al mismo tiempo que las funciones de las instituciones educativas se orientan a un ideal de progreso, los jóvenes depositan en éstas sus posibilidades de desarrollo (3). En este contexto, el mercado laboral demanda recursos humanos con determinados conocimientos, habilidades y destrezas que no siempre son los que los egresados adquieren en las instituciones educativas, de tal manera que el vínculo entre formación profesional, mercado e inserción laboral se establece en términos ambiguos, confusos e incluso discordantes (2).
Por otro lado, el actual mercado laboral no es ajeno al proceso de globalización, al desarrollo tecnológico y al contexto social, político y económico de un país. En los últimos años y bajo el discurso de desarrollo y crecimiento económico se han aprobado en México una serie de reformas estructurales que hoy en día determinan las reglas para insertarse al mercado de trabajo. Articuladas a lo que se denominó "Pacto por México", el Gobierno del último sexenio (20122018) se caracterizó por la implementación de una serie de "ambiciosas y regresivas" reformas estructurales en diversos ámbitos: materia laboral, energéticos, competencia económica, telecomunicaciones y radiodifusión, educación, entre otras (4). Particularmente la reforma laboral que "se publicitó como indispensable para la creación de empleo estable y bien remunerado al flexibilizarse el mercado de trabajo y las relaciones laborales" (5), provocó inestabilidad, desprotección y vulnerabilidad en las y los trabajadores. Como consecuencia de estos cambios, las profesiones de la salud, entre ellas enfermería, hoy en día se encuentran sumergidas en procesos laborales 
con condiciones precarias y poco favorables para la fuerza de trabajo (6).

La Real Academia Española (7) define "expectativa" como la esperanza de realizar o conseguir algo o la probabilidad razonable de que algo suceda. Para Vroom (8), una expectativa es la creencia de que una conducta específica producirá un resultado específico. Por su parte, Portal (9) la define como la esperanza o probabilidad de lograr una meta particular que se caracteriza por ser cambiante y depender de factores personales y sociales que pueden modificarla. De acuerdo con Lewin y Tolman, citados por Varela (10), una expectativa es "la valoración subjetiva de la posibilidad de alcanzar un objetivo particular". Para fines de este trabajo, "expectativa" se define como la esperanza subjetiva y cambiante de lograr una meta particular o un efecto deseado que se proyectará a futuro. En este sentido, las expectativas se van construyendo a través de la vida y tienen una influencia directa con el contexto en el que los individuos se desarrollan.

Es evidente que las expectativas en el ámbito educativo se conciben como posibilidades razonables y sostenidas de que algo ocurra en situaciones propiamente académicas (11), es decir, representan aquello que los estudiantes esperan realizar a partir y alrededor de su vida formativa. Por otra parte, las expectativas laborales son posibilidades que se esperan conseguir al terminar los estudios profesionales (12). Para Guzmán (12) las expectativas laborales requieren de un conocimiento o un imaginario previo sobre los posibles espacios en los cuales se puede trabajar, cumplir y desarrollar los conocimientos, habilidades y destrezas que se adquirieron durante la formación académica.

Debe señalarse, que la práctica de enfermería históricamente se ha caracterizado por desarrollarse profesionalmente en el área asistencial, particularmente en hospitales de segundo y tercer nivel de atención. Hasta 2016, en el sistema de salud en México se tenían registrados a nivel nacional un total de 268. 014 enfermeras y enfermeros (13). El Instituto Nacional de Estadística y Geografía (INEGI) reportó en 2015 que 93 de cada 100 profesionales de enfermería laboraban en los servicios de atención a la salud y asistencia social, los otros siete se dedicaban a actividades administrativas, gubernamentales, servicios financieros, comercio y otras (14). Para ese mismo año, 76 de cada 100 enfermeros y enfermeras trabajaban en el sector público y el resto en el sector privado ${ }^{1}$. La práctica de enfermería también se ha distinguido profesionalmente por brindar cuidados básicos en el domicilio de los pacientes, situación que ha generado un aumento de empresas privadas de cuidadoras/es en varios estados del país en los últimos años, siendo el Estado de Nuevo León en donde se concentra el mayor número de empresas privadas de este tipo seguido de la Ciudad de México y Puebla (15).

Lo más significativo, es que a partir de los grandes avances teóricos- disciplinares y profesionales que ha tenido la enfermería en las últimas décadas, se ha enfatizado en la necesidad de ampliar los horizontes de aplicación del conocimiento (16) a través de asumir una práctica profesional que traspase las fronteras de la atención hospitalaria y del sector público, abriendo así, posibilidades de ejercicio independiente de la profesión y fomentando otros campos de desarrollo disciplinar como la investigación, la gestión y la docencia.

El objetivo de esta investigación fue conocer las expectativas académicas y laborales de estudiantes de enfermería que cursaban elúltimo semestre de la licenciatura en enfermería en una institución educativa de la Ciudad de México, que se caracteriza por reportar anualmente el mayor número de egresados en la entidad ${ }^{2}$ . La relevancia de la investigación radica en la escasez de estudios que abordan las expectativas académicas y laborales en estudiantes de enfermería. Aunque existen estudios sobre el tema a nivel nacional e internacional, la mayoría de ellos se centran en estudiantes de disciplinas distintas a la enfermería $(9,11-12,18$ 25), en las expectativas que los estudiantes tienen de las instituciones educativas $(26,27)$, los programas académicos (28) y los docentes (29). Igualmente, se encauzan hacia las expectativas que los profesores tienen hacia los estudiantes (30-32), así como de las que existen entre estudiantes y profesores de acuerdo a los roles académicos que desempeñan (33).

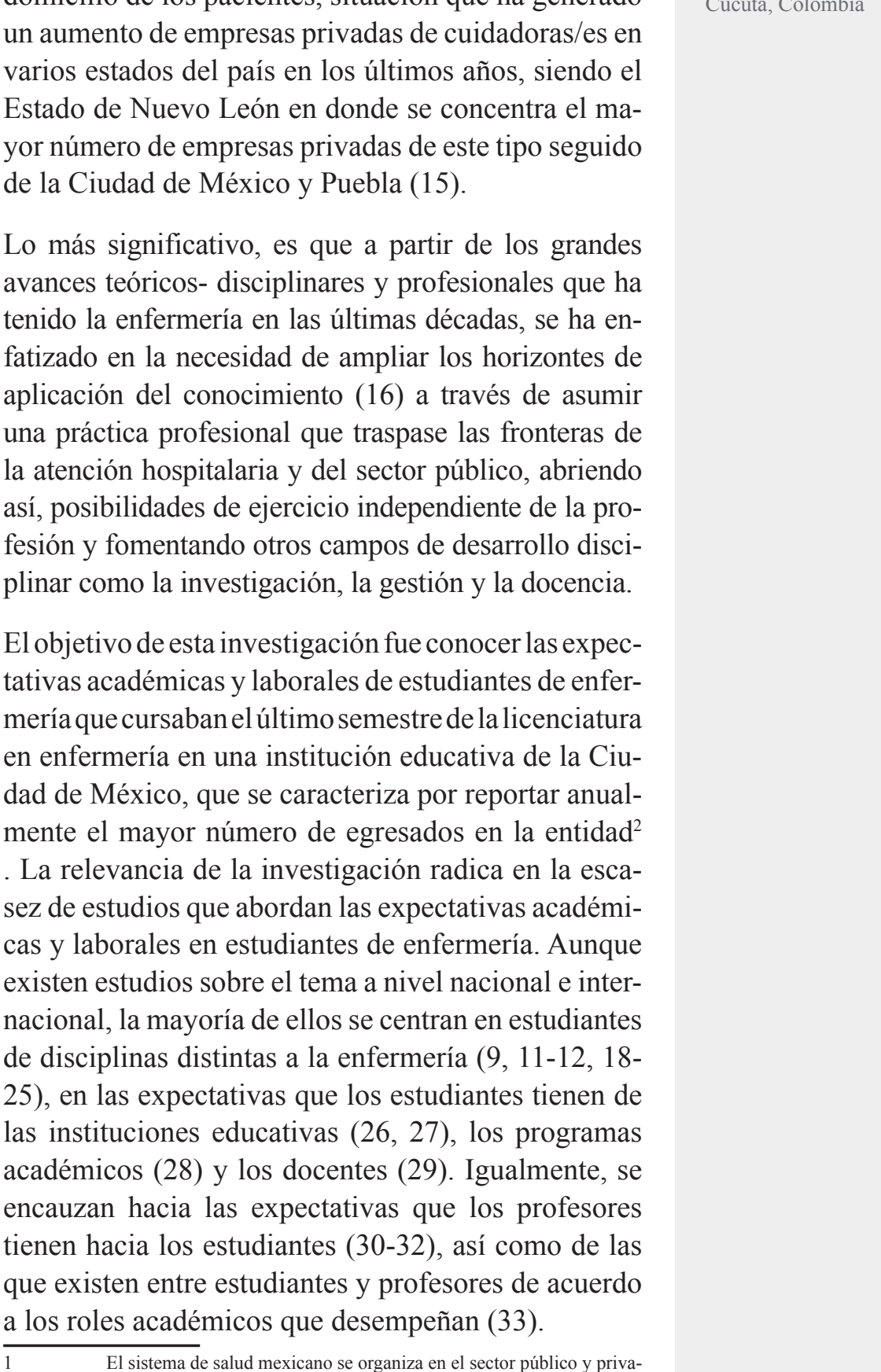


ISSN-PRINT

1794-9831

E-ISSN 2322-7028

Vol. 16 No. 2

May - Ago 2019

Cúcuta, Colombia
Dar cuenta de lo que los estudiantes de enfermería esperan una vez que concluyan sus estudios profesionales proporciona evidencias empíricas para adecuar, incorporar y/o modificar uno de los elementos académicos de mayor relevancia en los procesos formativos, el plan de estudios. Esta investigación también representa una oportunidad para conocer la congruencia entre lo que esperan los futuros profesionales y los escenarios de trabajo que actualmente ofrece el mercado laboral en México, un aspecto que nos lleva a reflexionar sobre el grado de conocimiento de los estudiantes en torno a las reformas estructurales que han tenido lugar en el país en los últimos años.

\section{Objetivos}

\section{Objetivo General}

Conocer las expectativas académicas y laborales de los estudiantes que cursan octavo semestre de la Licenciatura en enfermería y Licenciatura en enfermería y obstetricia de una universidad pública de la Ciudad de México.

\section{Objetivos específicos}

- Describir las expectativas laborales y académicas de los estudiantes.

- Establecer la frecuencia de las expectativas académicas y laborales.

\section{Materiales y métodos}

Se realizó un estudio cuantitativo, descriptivo- exploratorio y transversal durante el ciclo escolar 2016-2. La población de estudio se integró por 492 estudiantes de ambos sexos, mayores de 18 años e inscritos en el último semestre de alguna de las dos carreras que se ofertan en la institución: Licenciatura en Enfermería (LE) y Licenciatura en Enfermería y Obstetricia (LEO). A partir de un diseño de muestreo probabilístico aleatorio simple se obtuvo una muestra de 105 estudiantes con un intervalo de confianza de 0,95.

Después de una búsqueda exhaustiva en la literatura nacional e internacional y al no encontrar ningún instrumento que midiera las expectativas académicas y laborales en estudiantes universitarios, se elaboró un cuestionario de autoadministración con preguntas cerradas. La primera versión del instrumento se sometió a una validación de contenido por parte de dos expertos. Después de realizar las respectivas adecuaciones, se aplicó una prueba piloto a 10 estudiantes de octavo semestre inscritos en el ciclo escolar 2016-2, en la que participaron 5 alumnos de la LE y 5 de la LEO. La versión final del instrumento quedó integrada por 24 preguntas de opción múltiple estructurada en tres secciones: en la primera se recolectó información sobre las características sociodemográficas y las condiciones académicas y laborales de la población de estudio (10 preguntas); en la segunda se recogió información sobre las expectativas académicas (6 preguntas) con cuestiones sobre lugar para realizar servicio social, opción de titulación a elegir, planes profesionales, realización de estudios de posgrado, por mencionar algunas; y, en la tercera, sobre las expectativas laborales ( 8 preguntas) con preguntas encaminadas a conocer área disciplinar, sector y lugar para trabajar, categoría laboral, sueldo, entre otras.

La aplicación del instrumento se llevó a cabo en las instalaciones de la institución educativa de mayo a junio de 2016. Para el procesamiento de la información se utilizó el paquete estadístico IBS SPSS Statistics versión 20 y para el análisis se calcularon frecuencias absolutas y relativas.

Por último, con respecto a las cuestiones éticas se consideró la Declaración de Helsinki especialmente el principio de protección de "la vida, la salud, la dignidad, la integridad, el derecho a la autodeterminación, la intimidad y la confidencialidad de la información de las personas que participan en la investigación" (37). Después de darles a conocer los objetivos y las características de la investigación, los universitarios colaboraron de manera voluntaria, a quienes se les garantizó el anonimato y el uso de datos sólo para fines investigativos. El estudio fue aprobado por el Comité de Ética e Investigación de la institución donde se realizó.

\section{Resultados}

En el estudio participaron 105 estudiantes, 71.4\% mujeres y $28.6 \%$ hombres. La edad que predominó entre los participantes fue de 23 años (28.6\%). Sobre su estado civil, $87.6 \%$ afirmó ser solteros y sólo $19.0 \%$ mencionó tener hijos.

El $64.8 \%$ de los participantes cursaba la LEO y $35.2 \%$ la LE; el $53.3 \%$ estaba inscrito en el turno vespertino y $46.7 \%$ en el matutino; $65.7 \%$ inició sus estudios de licenciatura en el año 2012, 22.9\% 
en $2013,2,9 \%$ en 2011 y únicamente el $1.0 \%$ en 2008, el 7.5\% no contestó esta pregunta. En relación con su forma de ingreso a la licenciatura ${ }^{3}$ , 68.6\% señaló haber entrado por pase reglamentado, $27.6 \%$ mediante examen de admisión y $3.8 \%$ por cambio de carrera. El $82.9 \%$ mencionó no tener trabajo mientras que el $17.1 \%$ manifestó estar trabajando en el momento en el que se recolectó la información; para el $50.0 \%$ la actividad laboral estaba muy relacionada con su formación académica, el $16.6 \%$ de los casos tenía poca relación y el $33.3 \%$ no tenía ninguna relación.

\section{Expectativas académicas}

La mayoría de los estudiantes espera realizar el servicio social ${ }^{4}$ en hospitales de segundo y tercer nivel de atención: $43.8 \%$ en un hospital general ${ }^{5}, 17.1 \%$ en un Instituto $\mathrm{Nacional}^{6}, 11.4 \%$ en un hospital de especialidad, $3.8 \%$ en actividades de investigación en una institución educativa u hospitalaria y $2.9 \%$ en alguna institución de salud de primer nivel de atención (clínica de medicina familiar o centro de salud). Al $17.1 \%$ le gustaría participar en un programa comunitario en alguna provincia del país ${ }^{7}$, el $1.0 \%$ indicó querer solicitar la liberación del servicio social por tener antigüedad laboral en una institución pública de salud y $2.9 \%$ de los estudiantes aún no tenían claro dónde realizar el servicio.

Como opción de titulación, $29.5 \%$ de los estudian-

3 Las formas de ingreso a nivel licenciatura establecidas en el Reglamento de la institución educativa son: 1. Pase reglamentado, 2. Concurso de selección por medio de un examen de conocimientos y, 3. Cambio de carrera. La primera sólo es opción para los egresados de cualquiera de los planteles de la Escuela Nacional Preparatoria (ENP) y del Colegio de Ciencias y Humanidades $(\mathrm{CCH})$ pertenecientes a la UNAM siempre y cuando hayan concluido el bachillerato en un plazo no mayor a cuatro años y con un promedio general de siete o más. La segunda está disponible para todas aquellas personas que cuenten con estudios de bachillerato y promedio mínimo de siete. La última opción se refiere al trámite que solicita el estudiante que está inscrito en una carrera y quiere cambiarse a otra dentro del mismo o diferente plantel (38)

4 Se entiende por servicio social el conjunto de actividades de carácter obligatorio y temporal que realizan y prestan los estudiantes universitarios en interés de la sociedad y el Estado, como requisito para obtener el título o grado académico correspondiente; forma parte de los planes y programas de estudios y complementa su formación académica, contempla tres dimensiones: formativa, social y retributiva (39).

5 Los hospitales generales en México pertenecen al segundo nivel de atención, en ellos se brinda esencialmente atención ambulatoria y de hospitalización en las cuatro principales especialidades médicas: medicina interna, cirugía general, pediatría y ginecoobstetricia.

6 Los Institutos Nacionales se caracterizan por ofrecer atención especializada correspondiente al tercer nivel de atención, por tanto, reciben a los pacientes referidos de los dos niveles previos y de los servicios de emergencia. Actualmente, la Secretaría de Salud cuenta con doce Institutos Nacionales centrados en las siguientes especialidades: cardiología, pediatría, perinatología, nutrición, psiquiatría, cancerología, de enfermedades respiratorias, neurología, rehabilitación, medicina genómica, geriatría y salud pública.

7 Una de las opciones de servicio social para los alumnos es la incorporación a programas de atención comunitaria en diversas entidades del país. tes prefieren realizar estudios de posgrado, $18.1 \%$ elaborar tesis o tesina, $17.1 \%$ trabajar en un Proceso de Atención de Enfermería (PAE) y 15.2\% presentar el Examen Profesional por Áreas de Conocimientos (EPAC). Entre las opciones de menor interés se encuentra la ampliación y profundización de conocimientos mediante cursos o diplomados $(9.5 \%)$, actividades de investigación (2.9\%) y actividad de apoyo a la docencia (2.9\%). El 2.9\% respondió no saber en ese momento por qué opción titularse y $1.9 \%$ indicó otra opción para realizar el trámite de titulación ${ }^{8}$.

Al terminar la carrera, el $61.9 \%$ de los estudiantes espera trabajar y al mismo tiempo estudiar una especialidad, $4.8 \%$ considera trabajar y estudiar una maestría, $13.3 \%$ quisiera exclusivamente trabajar y $3.8 \%$ únicamente cursar una especialidad; al 15.2\% les gustaría estudiar una segunda carrera y el $1.0 \%$ no contestó a la pregunta.

Del total de estudiantes a quienes les interesa realizar estudios de posgrado (70.4\%), 45.9\% desea estudiar en el extranjero, $28.3 \%$ en provincia, $20.2 \%$ en la ciudad de México, $1.3 \%$ tanto en el extranjero como en provincia y $4.0 \%$ no respondió.

En relación con el grado en que los estudiantes consideran que su formación les dio suficientes conocimientos teórico- prácticos para integrarse al área laboral de su preferencia, el $10.5 \%$ señaló que fue muy alto, $59.0 \%$ lo considera alto, al $28.6 \%$ le pareció regular, $1.0 \%$ dijo que fue bajo y $1.0 \%$ no respondió la pregunta.

\section{Expectativas laborales}

Respecto al área a la que esperan integrarse a trabajar, el $63.4 \%$ de los estudiantes señaló tener preferencia por el ámbito asistencial, $16.1 \%$ por el administrativo, $9.5 \%$ por el docente y $8.5 \%$ por el de investigación; sólo el $0.9 \%$ quisiera desarrollarse en el área asistencial simultáneamente con la docencia y el otro $0.9 \%$ con la administración.

De igual manera, el $84.7 \%$ de los alumnos aspira a laborar en el sector público y $8.5 \%$ en el privado; sólo el $5.7 \%$ quisiera trabajar por cuenta propia y $0.9 \%$ desea laborar en el sector público y simultáneamente ejercer su práctica de manera independiente. Al terminar su servicio social, el $64.8 \%$ de los estudiantes quisiera incorporarse a trabajar dentro de los primeros seis meses, $26.0 \%$ de siete me-

\footnotetext{
De acuerdo con el Reglamento de la institución otras formas de titulación son: totalidad de créditos y alto nivel académico y trabajo profesional (40).
}

E-ISSN 2322-7028

Vol. 16 No. 2

May - Ago 2019

Cúcuta, Colombia 
ISSN-PRINT

1794-9831

E-ISSN 2322-7028

Vol. 16 No. 2

May - Ago 2019

Cúcuta, Colombia ses a un año y $8.6 \%$ hasta después de un año.

De modo similar, al 92.3\% de los participantes les interesa trabajar en segundo y tercer nivel de atención (Gráfica 1), al 52.3\% en hospitales generales, al $20.0 \%$ en hospitales de especialidades y al $20.0 \%$ en
Institutos Nacionales. Sólo 1.9\% se inclina por el primer nivel de atención en un centro de salud o clínica de medicina familiar; al 2.8\% le interesa trabajar en una institución de educación superior, 1.9\% prefiere laborar por cuenta propia y $0.9 \%$ quisiera combinar el primer y segundo nivel de atención.

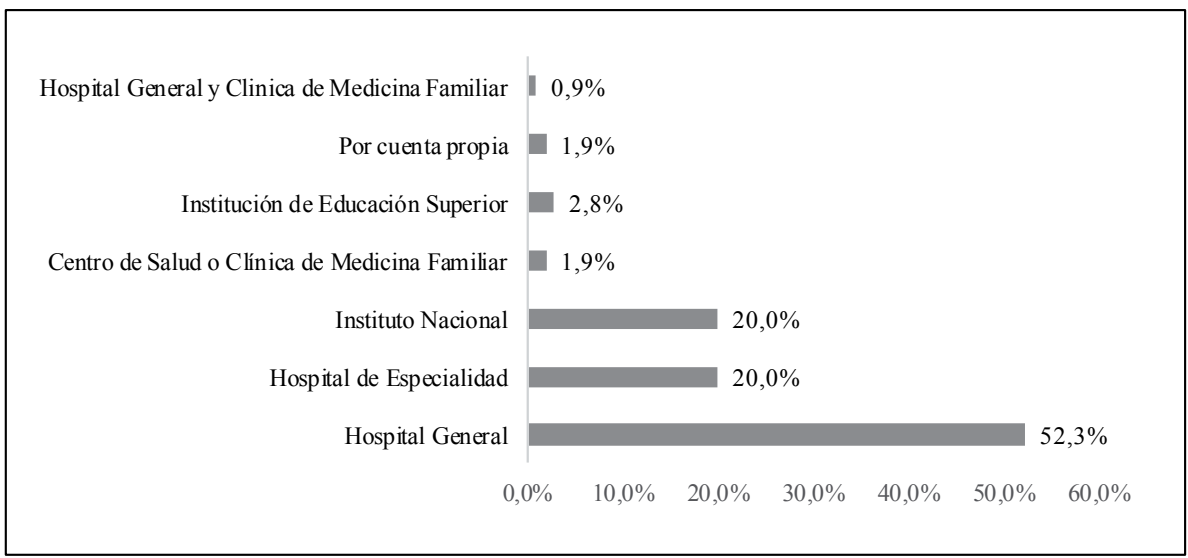

Gráfica 1. Expectativas del espacio laboral

Fuente: Elaboración propia a partir del Instrumento Expectativas académicas y laborales en estudiantes de enfermería (2016).

Cabe destacar, que el $57.1 \%$ espera incorporarse al ámbito laboral con una categoría de enfermero (a) especialista, 30.5\% como enfermero (a) general, $4.8 \%$ como docente, $3.8 \%$ como enfermero (a) auxiliar, $2.9 \%$ como investigador(a) y $0.9 \%$ como enfermero (a) obstetra. Durante el primer año de trabajo, el $57.1 \%$ de los universitarios espera recibir un salario mensual de $\$ 5,100.00$ a $\$ 8,000.00,31.4 \%$ de $\$ 8,100.00$ a $\$ 14,000.00$ y $11.4 \%$ de $\$ 3,400.00$ a $\$ 5,000.00$ pesos mexicanos.
Entre las principales prestaciones y condiciones laborales a las que aspiran acceder se encuentran: aguinaldo $(92.3 \%)$, vacaciones y prima vacacional (90.4\%), servicio médico (90.4\%), días de descanso fijo $(71.4 \%)$, licencia de maternidad o paternidad (59.0\%), contrato de tiempo indefinido (57.1\%) y la posibilidad de acceder a un crédito para vivienda (56.1\%). De menor relevancia están: los vales de despensa, días económicos, prestaciones relacionadas con riesgos de trabajo, entre otros (Tabla 1).

Tabla 1. Condiciones y prestaciones laborales

\begin{tabular}{lcc}
\hline \multicolumn{1}{c}{ Tipo de prestación } & Frecuencia & \% \\
\hline Aguinaldo & 97 & 92.3 \\
Vacaciones y prima vacacional & 95 & 90.4 \\
Servicio médico & 95 & 90.4 \\
Días de descanso fijos & 75 & 71.4 \\
Licencia de maternidad/ paternidad & 62 & 59.0 \\
Contrato por tiempo indefinido & 60 & 57.1 \\
Crédito para vivienda & 59 & 56.1 \\
Otros (días económicos, vales de despensa) & 2.0 & 1.9 \\
\hline
\end{tabular}

Fuente: Elaboración propia a partir del Instrumento Expectativas académicas y laborales en estudiantes de enfermería (2016). 
Al 40.9\% de los participantes les interesa quedarse a trabajar en la ciudad de México, mientras que 27.6\% quisiera hacerlo en el extranjero, $27.6 \%$ en alguna provincia mexicana y sólo al $3.8 \%$ en provincia y en el extranjero.

\section{Discusión}

En la población objeto de estudio predominó una mayor participación de mujeres en comparación con la de los hombres. De acuerdo con el SIARHE (41), en 2016 el 87.1\% del personal del sistema mexicano de salud eran mujeres y sólo $12.9 \%$ hombres. La enfermería ha sido una profesión históricamente atribuida y desempeñada predominantemente por mujeres, un fenómeno presente en la actualidad. En un estudio realizado en 2010 con alumnos de enfermería en dos sedes de la Universidad Nacional Autónoma de México (FES Zaragoza y FES Iztacala) se encontró que el $76.7 \%$ eran mujeres (42).

Es de hacer notar, que el perfil sociodemográfico de los participantes en esta investigación es similar a los reportados en otros estudiantes de enfermería (43) particularmente en lo relacionado con: la edad, el estado civil, la participación en el área laboral y el número de dependientes económicos. El promedio de edad de los y las estudiantes concuerda con el grado académico que cursaban los alumnos al momento de responder el instrumento, considerando que en México se inician estudios universitarios entre los $18 \mathrm{y}$ los 20 años (44).

La mayoría de los alumnos manifestaron ser solteros, sin hijos y dedicarse de tiempo completo a los estudios, condiciones favorables para una estabilidad y un mejor rendimiento académico y, además, constituyen factores primordiales de permanencia en la carrera (45) sobre todo, si se considera que en los últimos años se ha reportado un elevado índice de deserción en las escuelas de enfermería en América Latina, traducido en un número insuficiente de personal de enfermería en las instituciones de salud (43).

La preferencia por titularse a través de estudios de posgrado concuerda con los últimos reportes anuales sobre egresados de la institución educativa en la que se llevó a cabo la investigación (46). Con base en estos datos, los estudios de posgrado se ubican dentro de las tres primeras opciones elegidas por los alumnos. Es importante decir que, de los estudios de posgrado, las especialidades son las que tienen mayor demanda en comparación con los programas de maestría y doctorado. De acuerdo con Ramírez (47) la mayoría de las especializaciones ofertadas en las escuelas de enfermería se centran principalmente en el área hospitalaria (cuidados intensivos, pediatría, geriatría, instrumentista, enfermería materno infantil, etc.) acorde con la actual estructura del sistema de salud, cuyo objetivo primordial se enfoca en la atención de la enfermedad y no en la prevención desde la atención primaria y la protección de la salud; por tanto, es una estrategia limitada a corregir los problemas de saludenfermedad-atención que prevalecen en nuestro país. Aunque la mayoría de los estudiantes aspira simultáneamente a trabajar y estudiar una especialidad, un número considerable espera trabajar y estudiar una segunda carrera. Aún es frecuente encontrar estudiantes que ingresan a la licenciatura de enfermería con la aspiración inicial de cursar la carrera de medicina. Al respecto, González et al. (42) encontraron que de 600 estudiantes de enfermería sólo el 61.7\% habían elegido esta carrera como primera opción.

Es evidente el interés de los estudiantes por quedarse a laborar y estudiar en la Ciudad de México. Argumento que justifica el limitado deseo de estudiar posgrados en alguna provincia, porque responde a la lógica de considerar que las mejores posibilidades académicas se siguen concentrando en las grandes urbes. Aunque se ha incrementado la oferta educativa a nivel de posgrado en enfermería en diversas provincias del país, el mayor número de programas se sigue concentrando en la Ciudad de México, tal como ocurre en la Escuela Nacional de Enfermería y Obstetricia (ENEO) donde actualmente se ofertan 15 especialidades (48). Además, existen programas de posgrado que se ofrecen únicamente en instituciones educativas ubicadas en la Ciudad de México.

El desinterés de los estudiantes por laborar en el extranjero contrasta con los resultados encontrados por Rosales et al., (49) quienes reportan que los estudiantes quieren migrar a otros países por considerar que existen menos oportunidades de desarrollo profesional en el país. En México aún es limitado el conocimiento sobre el fenómeno migratorio; sin embargo, se reconoce que, aunque la migración es incipiente, las repercusiones futuras pueden afectar seriamente al sistema de salud y a sus instituciones (50).

Al igual que el servicio social, el ámbito clínico sigue $\overline{\text { E-ISSN 2322-7028 }}$

Vol. 16 No. 2

May - Ago 2019

Cúcuta, Colombia 
ISSN-PRINT

1794-9831

E-ISSN 2322-7028

Vol. 16 No. 2

May - Ago 2019

Cúcuta, Colombia siendo el lugar de preferencia de los estudiantes para desarrollar su práctica profesional. Aunque las cuatro áreas de enfermería (docencia, investigación, asistencia y gestión) están presentes en el plan curricular, las expectativas laborales de los estudiantes podrían responder a que la mayor carga académica se concentra en el área asistencial. En suma, la preferencia de los estudiantes por el área asistencial se limita al segundo y tercer nivel de atención más que por centros de salud y unidades de medicina familiar. Inclinarse por el ámbito clínico hospitalario también es reflejo de la manera en que se distribuyen los recursos humanos en salud en México. En un estudio realizado en 2016 por Nigenda et al., (51) se informó que en la mayoría de los estados de la República Mexicana se distribuye un mínimo porcentaje de personal de enfermería en unidades de primer nivel, siendo menor la asignación en la Ciudad de México, Jalisco, Campeche y Zacatecas.

Si bien es cierto que históricamente la formación y desarrollo profesional en enfermería se ha desarrollado en escenarios clínicos, sin embargo existe una preferencia por hospitales generales públicos, hospitales de especialidades e Institutos Nacionales como lugares para integrarse al ambiente laboral, porque se les vincula fuertemente con los planes de estudio y el perfil de egreso, ya que se prefiere la formación clínica hospitalaria y no tanto el primer nivel de atención y el trabajo comunitario. Asimismo, se observa, una clara inclinación de los estudiantes por elegir campos del área asistencial para realizar su servicio social, lo que refleja y define las expectativas laborales de los estudiantes. Es importante señalar que el mayor número de plazas de servicio social otorgadas por las instituciones de salud se ubican en segundo y tercer nivel de atención. En 2012 se designaron 3.867 plazas para hospitales de la Secretaría de Salud y para instituciones como el DIF Nacional con Programas de vacunación que sólo fueron 7 y 78 respectivamente (52).

Ante este panorama, áreas de desarrollo profesional como la investigación y el ejercicio libre de la profesión no figuran como opciones académicas y laborales para los estudiantes. Para fomentar estos nuevos escenarios es necesario incentivar en los futuros profesionales conocimientos, habilidades y herramientas para el desarrollo de competencias que les permitan ejercer su profesión en un marco de independencia y autonomía. Esto lleva a repensar los perfiles establecidos en los planes de estudio, así como su estructura.
En cuanto al sector laboral, el público es el de mayor preferencia. Actualmente el mayor número de plazas de enfermería ofertadas y ocupadas en el Sistema Nacional de Salud corresponden al sector público. Aunque las plazas ocupadas en el sector privado han ganado terreno en los últimos años; no obstante continúan siendo menores en comparación con el sector público. Tan sólo en 2018, la Secretaría de Salud registró 199. 302 enfermeras y enfermeros (53), para el mismo año se contemplaron 8.898 plazas de personal de enfermería a nivel privado (53). No obstante, a diferencia de lo que se ha registrado en otros estudios (54-55) es en el sector privado -incluido el ejercicio libre de la profesión- donde se insertan los recién egresados.

Respecto al tiempo para incorporarse al campo laboral, estudios como el de Juárez et al. (56) demuestran que los egresados consiguen un trabajo entre los seis primeros meses y hasta un año posterior al término de sus estudios, como esperan los entrevistados del presente estudio. Es conveniente indicar que, en muchos casos, los pasantes de servicio social son invitados a presentar su solicitud para integrarse a trabajar en las mismas instituciones dónde realizaron su pasantía.

Existe gran expectativa entre los estudiantes por incorporarse al sector laboral con una categoría de enfermero (a) especialista. Sin embargo, para aspirar a una plaza en México con esta categoría se requieren estudios de posgrado. El interés por ser contratados(as) como especialistas, particularmente en alumnos que cursaban la LEO, responde a una confusión al considerar a la obstetricia como una especialidad y no como parte de su formación de pregrado. Por otro lado, las actuales condiciones laborales en el país dificultan el ingreso para trabajar en alguna institución de salud pública o privada, con una categoría de especialista, aun teniendo el grado. Desde esta perspectiva, Müggenburg y colaboradores (54-55) muestran que las tres principales categorías con las que los estudiantes se integran al mercado laboral son, en orden, auxiliar de enfermería, enfermero (a) general y licenciado (a). Otros estudios (55) señalan que la principal categoría de inserción laboral es como enfermero (a) general y enfermero (a) auxiliar. Un estudio realizado en la ENEO (57) muestra que los egresados de la generación 2003 - 2006 se incorporaron primero como enfermeros generales y auxiliares y posteriormente con la categoría de licenciados. La licenciatura en enfermería y obstetricia no figura como categoría 
laboral en la mayoría de las instituciones de salud. Como lo señala Pérez (57) la práctica de enfermería obstétrica en las instituciones hospitalarias no es aún una práctica aceptada de manera uniforme y generalizada, esto se refleja en las diversas categorías con las que se insertan los estudiantes al ingresar a trabajar. En este sentido, las expectativas de los estudiantes en relación con la categoría laboral distan con lo que se observa en el actual escenario laboral.

Con respecto a las expectativas de los estudiantes sobre el sueldo mensual que esperan recibir durante su primer año $(\$ 5,100.00 \mathrm{MXN}$ a $\$ 8,000.00 \mathrm{MXN})$ de trabajo, éste corresponde al promedio de ingreso del personal de enfermería en México, considerando que en el 2015 el INEGI reportó un ingreso de $\$ 50.70$ MXN por hora. Los resultados de esta investigación coinciden con Juárez y colaboradores (56) quienes afirman que para 2015 el salario percibido por los egresados en enfermería era de $\$ 4.001,00 \mathrm{MXN}$ quincenales ${ }^{9}$.

Por otra parte, Müggenburg et al. (54) indicaron que el $70 \%$ de los participantes recibían prestaciones laborales dentro de las que se destacan: seguro médico, préstamos, estímulos económicos, despensa, actualización técnica y actualización humanística. En esta investigación los estudiantes esperan recibir una serie de prestaciones que, como consecuencia del proceso de flexibilización del trabajo debido a las reformas estructurales, cada vez es más difícil que se incluyan en los actuales contratos laborales (58). Los cambios de estas reformas también implican que sea más accesible encontrar empleos eventuales o de medio tiempo, sin ningún tipo de estabilidad salarial o laboral, con estrategias de subcontratación y poca remuneración, ajenas a un sistema de prestaciones (3). Sobre estas realidades la inquietud principal de los estudiantes se centra en el reconocimiento de estos procesos de cambio y sus implicaciones para aquellos que aspiran a una formación profesional ${ }^{10}$. No obstante, aún

9 En 2018 el Observatorio Laboral de la Secretaría del Trabajo y Previsión Social determinó que el salario mensual promedio para enfermería a nivel licenciatura es de $\$ 9.311,00 \mathrm{MXN}$ (59).

10 Resulta interesante esperar cómo será el panorama en materia laboral en México con la actual administración federal (2018 - 2024) con una nueva pro- se requieren estudios que profundicen en las actuales condiciones laborales del personal de enfermería en México.

Aunque Rosales et al., (49) reportaron expectativas de migración en estudiantes de enfermería a causa de los bajos salarios en el país, es probable que el limitado interés de los participantes en este estudio por trabajar en el extranjero o en alguna provincia de México se deba a la concentración de recursos e infraestructura de atención a la salud en zonas urbanas, como es la Ciudad de México.

\section{Conclusiones}

- Las expectativas académicas y profesionales de los estudiantes que participaron en esta investigación se centran en el ámbito clínico, principalmente en hospitales de segundo y tercer nivel de atención. Áreas profesionales como la docencia, la gestión y la investigación no son campos prioritarios para los alumnos, así como tampoco el ejercicio libre de la profesión. Como se ha visto en las últimas décadas, los futuros egresados esperan desarrollarse profesionalmente en espacios urbanos y en el sector público.

- A partir de estos resultados consideramos pertinente conocer la influencia que tienen los planes de estudio sobre los futuros profesionistas, particularmente sobre lo que esperan en el ámbito laboral y académico. Desde las instituciones educativas se requiere un profundo análisis para elaborar y redefinir planes de estudio congruentes con el contexto actual y las posibilidades laborales y de desarrollo profesional, así como repensar los perfiles de egreso en las instituciones educativas en las que se oferta la Licenciatura de Enfermería en México.

\section{Conflicto de interés}

Las autoras declaran no tener conflictos de interés.

puesta de reforma laboral que pretende mejorar los salarios, eliminar la subcontratación y trabajar en la justicia laboral, por mencionar algunos aspectos.
E-ISSN 2322-7028

Vol. 16 No. 2

May - Ago 2019

Cúcuta, Colombia 
ISSN-PRINT

$1794-9831$

E-ISSN 2322-7028

Vol. 16 No. 2

May - Ago 2019

Cúcuta, Colombia

\section{Referencias Bibliográficas}

1. Sánchez M, Cárdenas L, Carmona B. La formación y el ejercicio profesional en enfermería. En: Vázquez Martínez D, Cuevas Álvarez L, Crocker Sagástume R. La formación de personal en México. México: Programa colaborativo de Recursos Humanos en Salud; 2005. p. 45-76.

2. Paz Rodríguez F, Betanzos Díaz N, Uribe Barrera N. Expectativas laborales y empleabilidad en enfermería y psicología. Aquichan [Internet]. 2014 [Consultado Junio 1 de 2018]; 14(1):67-78. Disponible en: http://www.redalyc.org/articulo.oa?id=74130041007

3. Pérez Ruiz A. Desigualdad, mercado laboral y educación superior en América Latina. El Cotidiano [Internet]. 2012 [Consultado Junio 1 de 2018]; (176):47-55. Disponible en: http://www.redalyc.org/articulo. oa? id $=32525230005$

4. Alzaga Sánchez O. Las iniciativas de reforma laboral de 2016 y el antecedente de 2012. Alegatos 2016; (93):435-446.

5. Anguiano Orozco A, Ortiz Magallón R. Reforma Laboral en México: precarización generalizada del trabajo. El Cotidiano [Internet]. 2013 [Consultado Junio 1 de 2018]; (182):95-104. Disponible en: http:// www.redalyc.org/articulo.oa?id $=32529942010$

6. Jarillo Soto EC, Outón Lemus M, Salinas Urbina AA. Formación y práctica de profesionales de la salud: una mirada a su historia en México. En: Chapela Mendoza MC, Contreras Garfias ME. La Salud en México. Serie del Bicentenario. México: Universidad Autónoma Metropolitana Xochimilco; 2011. p. 221-245.

7. Diccionario de la Lengua Española (DEL). Expectativa. Real Academia Española, Asociación de Academias de la Lengua Española. [Internet]. 2016 [Consultado el 3 de Agosto de 2016]. Disponible en: http://dle.rae.es/?id=HI1X80V

8. Vroom VH. Work and Motivation. New York: Wiley; 1964.

9. Portal Cavero KA. Expectativas del ejercicio médico profesional en los internos de medicina del Hospital Nacional Arzobispo Loayza, 2015. [Tesis]. Lima: Universidad Nacional Mayor de San Marcos; 2015.

10. Varela Juárez RA. Administración de la compensación. Sueldos, salarios y prestaciones. México: Pearson; 2006.

11. Carrillo Olivera A, Ramírez Martínez JS. Expectativas académicas y laborales de estudiantes próximos a egresar de una licenciatura en Psicología Educativa. [Tesis]. Ciudad de México: Universidad Pedagógica Nacional; 2011.

12. Guzmán Torres J. Expectativas laborales de los estudiantes de la licenciatura en Administración Educativa, en la Universidad Pedagógica Nacional, Unidad Ajusco, ciclo escolar 2002 - 2003. [Tesis]. Ciudad de México: Universidad Pedagógica Nacional; 2006.

13. Secretaría de Salud, Sistema de Información Administrativa de Recursos Humanos en Enfermería (SIARHE). Numeralia. Recursos. [Internet]. 2017 [Consultado el 2 de Septiembre de 2017]. Disponible en: http://www.salud.gob.mx/unidades/cie/siarhe/?Id_URL=numeralia\&_Tipo=recursos\&_Id=2

14. Instituto Nacional de Estadística y Geografía (INEGI). Estadísticas a propósito de... Día internacional de la enfermera y el enfermero (6 de enero). [Internet]. 2015 [Consultado el 2 de Septiembre de 2017]. Disponible en: http://www.inegi.org.mx/saladeprensa/aproposito/2015/enfermera0.pdf

15. Zúñiga N. Aumenta en México enfermería a domicilio. Periódico Reforma, 15 de Julio de 2015.

16. Arenas Montaño G, Robles Mendoza AL, Santillán M, La práctica privada en Enfermería. Aspectos psicológicos, administrativos, éticos - legales y de género. México: Universidad Nacional Autónoma de México. Facultad de Estudios Superiores Iztacala; 2014.

17. López Arellano O, Jarillo Soto EC. La reforma neoliberal de un sistema de salud: evidencia del caso mexicano. Cad Saúde Pública 2017; 33, Sup. 2, 1-13. 
18. Botey Valles J, Deu Baigual E, Vilaplana Lapena E, Villanueva Margalef M. Expectativas profesionales de los alumnos de la sección de Ciencias Sociales de la E.U.F.P. San Gucat. Rev Interuniversitaria de Formación del Profesorado. III Seminario Estatal de Escuelas Universitarias de Magisterio 1988; (2):227-231.

19. Ramírez M. Expectativas profesionales de internos de medicina y su inclinación por la atención primaria de salud. An. Fac. med. [Internet]. 2008 [Consultado Junio 1 de 2018]; 69(3):176-181. Disponible en: http://www.scielo.org.pe/scielo.php?script=sci arttext\&pid=S1025-55832008000300006\&lng=es.

20. Borracci R, Pittaluga R, Manente D, Giorgi M, Rubio M. Expectativas de los estudiantes de medicina de la Universidad de Buenos Aires sobre su práctica profesional. Medicina (B. Aires) [Internet]. 2009 [Consultado Junio 1 de 2018]; 69(6):607-611. Disponible en: http://www.scielo.org.ar/scielo. php?script $=$ sci arttext\&pid=S0025-76802009000700003\&lng=es.

21. Hernández Ruiz A, Martín Llaguno M, Beléndez Vázquez M. Expectativas profesionales de estudiantes de Publicidad: ¿una profesión sexualmente sesgada? Un estudio piloto realizado con estudiantes de Alicante, Murcia y Navarra. Área Abierta 2012; 12(1):1-15.

22. Barraza Macías A, Gutiérrez Rico D. Las expectativas de inserción laboral de los alumnos de la licenciatura en Intervención Educativa. Investigación Educativa Duranguense, Universidad Pedagógica de Durango (8):25-40.

23. Gomariz Vicente MA, Fuentes Pérez P, Ayala de la Peña A. Aproximación comparada a las expectativas formativas y laborales de los estudiantes de grado de la facultad de Educación. Investigación e Innovación Educativa al servicio de Instituciones y Comunidades Globales, Plurales y Diversas. Actas del XVI Congreso Nacional/ II Internacional Modelos de Investigación Educativa de la Asociación Universitaria de Investigación Pedagógica (AIDIPE). Alicante 4 - 6 de Septiembre, 2013;648-658.

24. Hernández Hernández G, Fernández Pérez J. Expectativas profesionales: un estudio de caso. Rev. Mex. Orient. Educ. 2010; 7(19):18-27.

25. Ramírez Elizalde MR. Motivación y expectativas en estudiantes del último semestre de la licenciatura de Psicología Educativa de la UPN. [Tesis]. Ciudad de México: Universidad Pedagógica Nacional; 2008.

26. Pichardo Martínez MC, García Berbén AB, De la Fuente Arias J, Justicia F. El estudio de las expectativas en la universidad: análisis de trabajos empíricos y futuras líneas de investigación. REDIE Revista Electrónica de Investigación Educativa [Internet]. 2007 [Consultado el 10 de Octubre de 2015]; 9(1). Disponible en: http://redie.uabc.mx/redie/article/viewFile/153/263

27. Zysberg L, Zisberg A, Nursing students 'expectations of the college experience. Journal of Nursing Education 2008; 47(9):389-395.

28. Davis S, Schrader V. Comparison of syllabi expectations between faculty and students in a baccalaureate nursing program. Journal of Nursing Education 2009; 48(3):125-131.

29. Conejero Amorós ME, López Alacid M, Martínez Vicente AB. Expectativas del alumno hacia el profesor. Su influencia en el rendimiento académico. INFAD Rev. de Psicología International Journal of Developmental and Educational Psychology 2009; 2(1):411-418.

30. Mares Miramontes A, Martínez Llamas R, Rojo Sabaleta H. Concepto y expectativas del docente respecto de sus alumnos considerados con necesidades educativas especiales. RMIE 2009; 14(42):969-996.

31. Martínez Toro FJ. El rol de las expectativas docentes en los procesos de enseñanza-aprendizaje de la matemática. [Tesis]. Santiago de Chile: Universidad de Chile; 2015.

32. Valle Arias A, Núñez Pérez JC. Las expectativas del profesor y su incidencia en el contexto institucional. Rev. de Educación 1989; (90):293-319.

33. Yoder MK, Saylor C. Student and teacher roles: Mismatched Expectations. Nurse Educator 2002; 27(5): 201-203.

34. Universidad Nacional Autónoma de México. Agenda estadística UNAM 2017. Docencia. [Internet]. 2017 [Consultado el 18 de Diciembre de 2017]. Disponible en: http://www.planeacion.unam.mx/ Agenda/2017/disco/\#
1794-9831

E-ISSN 2322-7028

Vol. 16 No. 2

May - Ago 2019

Cúcuta, Colombia 
ISSN-PRINT

$1794-9831$

E-ISSN 2322-7028

Vol. 16 No. 2

May - Ago 2019

Cúcuta, Colombia

35. Universidad Autónoma Metropolitana. Anuario Estadístico 2016. Coordinación General de Información Institucional. Dirección de Planeación. Egresados. [Internet]. 2017 [Consultado el 18 de Diciembre de 2017]. Disponible en: http://www.transparencia.uam.mx/inforganos/anuarios/anuario2016/anuario_estadistico_2016.pdf

36. Instituto Politécnico Nacional. Estadística básica. Fin del ciclo escolar 2015-2016. Inicio de ciclo escolar 2016-2017. Secretaría de Gestión Estratégica. Dirección de Evaluación. [Internet]. 2017 [Consultado el 18 de Diciembre de 2017]. Disponible en: http://www.gestionestrategica.ipn.mx/Evaluacion/Documents/Estadistica/EBASICA_2016-2_V1.pdf

37. Asociación Médica Mundial. Declaración de Helsinki de la Asociación Médica Mundial [Internet]. 2008 [Consultado el 10 de Agosto de 2016]. Disponible en: http://www.wma.net/ es/30publications/10policies/b3/17c_es.pdf

38. Universidad Nacional Autónoma de México. Reglamento General de Inscripciones.

39. Proyecto de Norma Oficial Mexicana PROY-NOM-019- SSA2- 2012, Educación en salud. Utilización de campos clínicos para las prácticas clínicas y el servicio social de enfermería.

40. Escuela Nacional de Enfermería y Obstetricia. Universidad Nacional Autónoma de México. Lineamientos Generales para orientar las opciones de titulación carreras de licenciatura en enfermería y licenciatura en enfermería y obstetricia, sistema escolarizado, 2005.

41. Secretaría de Salud, Sistema de información Administrativa de Recursos Humanos en Enfermería (SIARHE). Numeralia. Recursos. [Internet]. 2016 [Consultado el 13 de Septiembre de 2016]. Disponible en: http://www.salud.gob.mx/unidades/cie/siarhe/?Id_URL=numeralia\&_Tipo=recursos\& $\underline{\mathrm{Id}=4}$

42. González Velázquez M, Lara Barrón A, Pineda Olvera J, Crespo S. Perfil de ingreso de los alumnos de Enfermería, Facultades de Estudios Superiores Iztacala y Zaragoza, UNAM. Enferm. univ 2014; 11(1):11-18.

43. De Valle Alonso MJ, Hernández López IE, Martínez Aguilera P, Barrón Cabrera SY, López Hernández M, Zúñiga Vargas ML. Perfil sociodemográfico y de intereses profesionales de los estudiantes de licenciatura en enfermería. Enferm. univ 2012; 9(1):32-34.

44. Torres Barrera S, Zambrano Lazárraga E. Breve historia de la educación de la enfermería en México. Rev Enferm Inst Mex Seguro Soc 2010; 18(2):105-110.

45. Plan de Acción Tutorial 2015- 2017, ENEO- UNAM, División de Estudios Profesionales. Programa Institucional de Tutoría.

46. Zarza Arismendi MD. -ENEO- Escuela Nacional de Enfermería y Obstetricia. Memoria UNAM [Internet]. 2015 [Consultado el 20 de Agosto de 2016]. Disponible en: http://www.planeacion.unam.mx/ Memoria/2015/PDF/5.1-ENEO.pdf

47. Ramírez Villegas RM. Enfermería. Retos para este nuevo siglo. Gaceta Urbana Migración y Salud 2007; (5):40-41.

48. Escuela Nacional de Enfermería y Obstetricia. Especialidades enfermería. [Internet]. 2018 [Consultado el 28 de Marzo de 2018]. Disponible en: http://www.eneo.unam.mx/posgrado/especialidades/ especialidades-enfermeria.php

49. Rosales Martínez Y, Nigenda G, Galárraga O, Ruíz Larios JA. Expectativas de migración internacional en estudiantes de enfermería en México. Salud Pública Méx 2010; 53(3):244-253.

50. Arroyo de Cordero G, Jiménez Sánchez J. Repercusiones de la escasez versus migración de enfermeras como fenómeno global. Rev Enferm Inst Mex Seguro Soc 2005; 13(1):33-40.

51. Nigenda G, Alcalde Rabanal J, González Robledo LM, Serván Mori E, García Saiso S, Lozano R. Eficiencia de los recursos humanos en salud: una aproximación a su análisis en México. Salud pública Méx 2016; 58:533- 542. 
52. Secretaría de Salud, Sistema de Información Administrativa de Recursos Humanos en Enfermería (SIARHE). Numeralia. Pasantes de enfermería en servicio social. Registro nacional de plazas. [Internet]. 2018 [Consultado el 28 de Marzo de 2018]. Disponible en: http://www.salud.gob.mx/unidades/ cie/siarhe/?Id_URL $=$ numeralia\&_Tipo=pasantes\&_Id $=1$

53. Secretaría de Salud, Sistema de Información Administrativa de Recursos Humanos en Enfermería (SIARHE). Numeralia. Recursos. [Internet]. 2018 [Consultado el 28 de Marzo de 2018]. Disponible en: http://www.salud.gob.mx/unidades/cie/siarhe/?Id_URL=numeralia\&_Tipo=recursos\&_Id=1

54. Müggenburg Rodríguez MC, Pérez Cabrera I, Ramírez Toríz EL, Castañeda Godínez MC. Seguimiento de egresados de la licenciatura de la ENEO - UNAM formación y desempeño laboral (2003-2006). Enferm. univ 2008; 5(1):21-29.

55. Müggenburg Rodríguez MC, Barreto Plácido F, García Ortiz MR. Seguimiento de egresados de la licenciatura en enfermería de la ENEO en 2000 a los tres años (2003). Enferm. univ 2006; 3(1):5-13.

56. Juárez Flores $C$, Báez Alvarado $M$, Hernández Vicente I, Hernández Ramírez M, Hernández Hernández O, Rodríguez Castañeda M. Opinión de egresados en enfermería sobre la congruencia de los contenidos curriculares con los requerimientos laborales. Enferm. univ 2015; 12(4)197-203.

57. Pérez Jiménez I. Proceso de iniciación de los alumnos de Enfermería, en la formación de las competencias obstétricas. Enferm. univ 2013, 10(3):75-83.

58. Nájera Nájera RM, Castrillón Agudelo MC, Manfredi M. Propuesta para el desarrollo y fortalecimiento de la enfermería en América Latina y el Caribe. México: Serie Académicos Universidad Autónoma Metropolitana; 2008.

59. Secretaría del Trabajo y Previsión Social. Observatorio Laboral. Reporte por carreras. Enfermería y cuidados. [Internet]. 2018 [Consultado el 28 de Marzo de 2018]. Disponible en: http://www.observatoriolaboral.gob.mx/\#/carrera/carrera-detalle nacional/5712/33/Enfermer $\% \mathrm{C} 3 \% \mathrm{ADa} \% 20 \mathrm{y} \% 20 \mathrm{cuida}-$ dos/Nacional/ 